\title{
S3440P Substitution in C-Terminal Region of Human Reelin Dramatically Impairs Secretion of Reelin from HEK 293T Cells
}

Elika Esmaeilzadeh-Gharehdaghi ${ }^{1}$, Ehsan Razmara ${ }^{1,2}$, Amirreza Bitaraf ${ }^{2,3}$, Mahdi Mahmoudi ${ }^{2}$, Masoud Garshasbi ${ }^{1 *}$

1. Department of Medical Genetics, Faculty of Medical Sciences, Tarbiat Modares University, Tehran, Iran

2. Rheumatology Research Center, Tehran University of Medical Sciences, Tehran, Iran

3. Department of Molecular Genetics, Faculty of Biological Sciences, Tarbiat Modares University

\section{*Corresponding author:}

Dr. Masoud Garshasbi

Department of Medical Genetics; Faculty of Medical Sciences; Tarbiat Modares University; Tehran, Iran,

Tel: +98-21-82884569, E-mail: masoud.garshasbi@ modares.ac.ir

Running Title: Human Reelin and CTR 


\begin{abstract}
Background: Reelin is a large extracellular glycoprotein secreted by Cajal-Retzius cells and has a main role during brain development, especially in neuronal migration. Reelin is comprised of N-terminal F-spondin like domain, eight tandem repeats, and a highly conserved basic Cterminal region (CTR). The CTR main role in the secretion of Reelin has been investigated by advertently inducing deletion in whole or a part of this region; however, the role of CTR point mutations on the secretion of Reelin is shrouded in mystery.
\end{abstract}

Materials and Methods: In this study, we performed experimental analyses on a subregion of Human Reelin containing 5th and 6th repeats (R5-R6), a part of 8 th repeat and the CTR which were amplified from cDNA of K562 and HEPG2 cells and cloned into a mammalian expressional plasmid (pVP22/myc-His). Bioinformatics investigation was performed on the CTR at both level of nucleotide and amino acid as well as mutant type. Random mutagenesis by errorprone PCR method was utilized to induce mutation in the CTR. The secretion efficiency of recombinant wild-type and mutant Reelin constructs compared in cell lysate and supernatant isolated from the transiently transfected HEK 293T cells using 6XHistag ELISA method.

Results: In-vitro study demonstrated that the CTR alteration(S3440P) leads to impairment of Reelin secretion even after overexpression.

Conclusions: Our results indicate that S3440P substitution in highly conserved structure of the CTR has an important effect on Reelin secretion.

Keywords: Reelin, CTR, Point Mutation, Secretion, RELN

\title{
Introduction
}


Reelin is a large extracellular glycoprotein (420-450 KD) which is secreted by Cajal-Retzius cells in the cerebral cortex of the brain (1). RELN gene encodes Reelin protein which has a key function in neuronal migration during the complex processes of the brain development (2). Reelin plays a pivotal role in different processes in synaptic plasticity, learning, and memory in the adult brain through interaction with NMDA receptor (3). Reelin is composed of three distinctive subdomains: the N-terminal containing F-spondin-like domain and signal peptide, eight Reelin repeats (RRs), and a short highly basic C-Terminal Region (CTR) (4). Each repeat of Reelin is subdivided by Epidermal-like Growth Factor (EGF) domain into two distinctive subdomains A and B (5). The EGF-like domains have important roles in protein-protein interactions, protein folding, and receptor binding (6). Mutations in EGF-like domains have a potential effect on protein secretion (7)

N-Terminal Region (NTR) is necessary for dimerization of Reelin during binding to specific receptors (8). The central fragment of Reelin involves third to sixth RRs which are sufficient for attaching to very low-density lipoprotein receptor (VLDLR) and Apolipoprotein receptor 2 (ApoER2) and leading to phosphorylation of adaptor protein Disabled-1 (Dab1) (9). C-Terminal Region (CTR) of Reelin composes less than 1\% of entire Reelin (32 AA of 3460 AA) (10). RELN gene consists of 65 exons and most part of CTR (from $\mathrm{Thr}^{3431}$ to the end ) is encoded by exon 65 (11). Exon 64 consists of 6 nucleotides and called as a micro-exon, this sequence is expressed in neuron cells but skipped by alternative splicing in other tissues (12). The main role of micro-exons has been revealed in various processes such as protein-protein interaction, generation of additional sites for post-translational modifications, and association with various neurological diseases (13). Alternative polyadenylation creates a short form of Reelin protein without the last 33 amino acids which are involving $10-25 \%$ of reelin mRNA in the mouse brain 
(12). Overexpression studies in COS-7 have shown that this truncated form of Reelin will be secreted much lower than full-length type, Reelin Wild-type (WT) (12). The primary sequence of CTR is highly conserved among vertebrates (Figure 1A) which could be justified by its essential physiological function (14). There is controversial evidence on the involvement of CTR in Reelin secretion; Although some studies have noted that the presence of CTR is essential for secretion, (15) others have shown that is not required. However, some studies have elucidated that CTR is only necessary for efficient transduction of downstream signaling $(14,16)$. Most of these publications have investigated the impact of presence or absence of CTR on Reelin secretion.

Effect(s) of CTR point mutations in the secretion of Reelin protein has not been revealed. Previously, production and secretion of reelin protein have been detected in supernatants and cell lysis of HEK 293T, Human Embryonic Kidney 293, by constructing the a subregion of the mouse reelin protein involving receptor binding domains (R5-R6) with or without (CTR) containing myc/6x his tag at the end of c-terminal (16),Furthermore it is demonstrated that Reelin mutations had same effect on secretion of full-length or a subregion of Reelin (17).

Here, we used cDNA derived from HEPG2 (human liver carcinoma cells) cells and random mutagenesis by error-prone PCR method for inducing mutation in the CTR. Eventually, a random mutation, c.10318T $>$ C, p. S3440P was detected in this highly conserved region and two vectors, containing the R5-R6 domains and a part of R8B domain of Human Reelin with either Wild-Type or Mutant forms of CTR (designated as R5-6/CWT and R5-6/CMUT respectively) were constructed meticulously.

The S3440P substitution in the CTR dramatically impaired secretion of the recombinant Reelin from HEK 293 T cells. 


\section{Material and Methods}

\section{Cells and Reagents}

In the present study, we utilized HEK 293T, K562 cells, and PRSV-ßgal plasmid (kindly gifted from Dr. Mehdi Banan, University of Social Welfare and Rehabilitation Sciences, Tehran, Iran), HEPG2 cells $\left(\right.$ ATCC $^{\circledR}$ HB-8065 ${ }^{\mathrm{TM}}$ ), Dulbecco's Modified Eagle's Medium -DMEM (Gibbco, united states), culture RPMI Medium 1640 (Gibco, United States), Fetal bovine serum (FBS) were obtained from Atlanta Biologicals, penicillin-streptomycin (Biosera, UK), HindIII (ER0501), EcoRI (ER0271), XbaI (ER0681) enzymes. E.coli Top10 and pVP22/myc-His2 plasmid- V485-01(were generously gifted by Dr. Azam Rahimpour, Shahid Beheshti University of Medical Sciences, Tehran, Iran), ßgal staining kite (Roche, Cat. No. 14159200), Lipofectamine2000 (Thermo Fisher Lot. No. 1857334), Gel purification kit (Roche, Cat. No. 11 732668 001), the PfuUltra High-Fidelity DNA Polymerase (Cat. No. 600380-51) were purchased from Agilent and GST 6XHis tag ELISA kite (ab128573), 6XHis tag antibody (ab9108), RNAX-plus (Cat. No. RN7713C), TAKARA-cDNA synthesis kit were applied during the experiment.

\section{Cell culture and Construction of Expression Plasmids}

HEPG2 cells were cultured in DMEM containing 10\% FBS and 1\% penicillin-streptomycin in a standard condition as in $5 \% \mathrm{Co}_{2}$ atmosphere and $37{ }^{\circ} \mathrm{C}$. Total RNA of the cells was isolated using RNAX-plus. The CTR fragment including coding region of the amino acids 3365-3460, harboring the 32 highly conserved amino acids of the Reelin CTR (3430-3460) was amplified 
from cDNA derived HEPG2 cells by following primers; CTR-Forward 5'aaagaattcGTCAACAACGGGATCAC-3',

CTR-Reverse

5 'aaatctagacgTGGGTATCGCCTAAGT-3'using a low fidelity enzyme and high concentration of $\operatorname{Mgcl} 2(3 \mathrm{mM}$ in total volume $25 \mu \mathrm{L})$. The pVP22/myc-His2 expressing plasmid was utilized for constructing the recombinant Human Reelin involving (R5-R6), a part of R4B, R8B and the CTR. VP22 gene is related to one of the structural proteins of herpes simplex virus type 1 (HSV1) and this gene was cut and replaced by CTR region by means of EcoRI/XbaI restriction enzymes. After affirming by Sanger sequencing, 5th and 6th repeats of Human Reelin protein were sub-cloned into constructs and ligated to CTR (wild-type and mutant) by using HindIII/EcoRI and appropriate ligating enzymes. This long fragment was comprised amino acids 1917-2673 of Reelin protein and amplified using PfuUltra High-Fidelity DNA Polymerase from cDNA derived from K562 cells by following primers; R5/R6-Forward-5' aaaaagcttaccatgGCCCAAACCAATGCTACA-3' and R5/R6-Reverse-5' aaaagaattcGGTGTCCAGCATAACGGTC -3'. The gene-specific sequences in the primers are bold and the remaining added as restriction or transcription initiation sites.

\section{Structures and Alignments}

CTR domain of Reelin was analyzed using ScanProsite (http://www.expasy.org/) and alignments of this region at the level of nucleotide sequences and amino acid residues were applied by using ClustalW (Clustalw, http://www.ebi.ac.uk/clustalw/) (Figure 1Aand B). Protein structure and the mutation impacts on protein flexibility and stability were analyzed by PyMOL (https://pymol.org/) and I-mutant V2.0 (https://www.folding.biofold.org/i-mutant/imutant2.0.html) after building the structure using the Phyre2 
(http://www.sbg.bio.ic.ac.uk/phyre2/) and SWISS-PROT (https://www.ebi.ac.uk/swissprot/) in a distinct PDB file. Figure 1C describes the amino acids substitution in CTR domain. We assessed our predicted structure accuracy by RAMPAGE online tool (https://www.mordred.bioc.cam.ac.uk/ rapper/rampage.php) that indicated almost $97 \%$ of residues in the most favored regions, around $2.1 \%$ of residues in allowed regions, and only $0.4 \%$ of residues in the outlier regions, which suggested that the modeled structures of RR8 and CTR were acceptable (Figure 1C).

\section{Cell culture and transfection}

HEK 293 T was cultured in DMEM, 10\% FBS, and 1\% penicillin-streptomycin in incubation condition involving 5\% CO2 and 95\% humidity. After reaching to 50-60\% confluency in T-25 flasks, the cells were transfected with $8 \mu \mathrm{g}$ of each vector- R5-6/CWT and R5-6/C MUTusing Lipofectamine $2000^{\circledR}$ reagent according to the manufacturer's instructions (Roche Biochemical, Germany). To control transfection efficiency, $\beta$-galactosidase ( $\beta$-Gal) reporter assay was applied.

\section{Enzyme-Linked Immunosorbent Assay (ELISA)}

We utilized the GST 6XHis tag ELISA kit and 6XHis tag antibody for evaluating the recombinant Reelin secretion and its intracellular level which was applied for 24 and 48 hours after transfection. All the reagents were applied based on standard protocols and standard curve was constructed by applying a serial dilution of the standard recombinant GST-6XHistag. This 
assay was performed in duplicate for all standard and test samples. The supernatants and cell lysates of non-transfected cells were used as negative control for this test.

\section{Statistical analysis}

Statistical analysis was applied by means of SPSS Version 25.0 (SPSS Inc., Chicago, IL, USA) and Graph-pad Prism v.6.0 (Graph Pad Software, San Diego, CA, USA). Continuous variables were presented as Mean value \pm SD for $n=3$ experiments. Analyzing results stemmed from the Cell line were applied based on the Tukey test and the variables were presented as Mean \pm SD.

\section{Results}

Reelin's CTR in both level of nucleotide sequences and amino acid residues is highly conserved (Figure 1A and B). The primary sequence of the CTR is completely conserved among all mammals, Figure 1A indicates this fact that 90 of 99 (90.9\%) nucleotide sequences encoding CTR are conserved among the species listed. CTR has highly positive charged peptides especially Arginine-rich domains. 12 out of 32 amino acids (38\%) are basic (Figure 1B), while none of them are acidic. Reelin's 8th repeat ended at $\mathrm{Val}^{3429}$ that leaves the last 32 amino acid residues for CTR (Figure 1B). All of the 32 comprising amino acid residues are conserved, except the first one in the crocodile, due to lack of micro-exon containing 6 nucleotides coding $\mathrm{Val}^{3429}$ and $\mathrm{Ser}^{3430}$. This evolutionary observation could suggest that the CTR has an important physiological function in vertebrates.

Given the controversial evidence about the role of CTR in the secretion of Reelin protein, in this study, we constructed two expressional plasmids (R5-6/CWT) and (R5-6/C MUT). To do so, the CTR fragment amplified from cDNA derived from HEPG2 cells and cloned. Several plasmids 
were sequenced, and clones containing wild-type sequence of CTR and a transition (c.10318T >C, p. S3440P) in the CTR (Figure 2) were identified. The long fragments harboring repeat 5 and 6 -RR6 and RR5 - of the Human Reelin were ligated to the upstream of CTR in both plasmids and Sanger sequencing did not show any other mutations in ORF of the two constructs.

In-silico prediction using I-Mutant2.0 (Predictor of Protein Stability Changes upon Mutations) showed that the S3440P substitution has a free energy change value (DDG) of -0.69 which predicts that this change reduces protein stability (Figure 1C); therefore, it was hypothesized that the secretion of Reelin protein could be altered by this substitution.

The secretion efficiency of the wild-type and mutant recombinant Reelin were compared in HEK $293 \mathrm{~T}$ cells. Cell lysates and supernatants were collected at different time points after transfection (Figure 2C) and measured with GST 6XHis tag ELISA kit utilizing 6XHis tag antibody. The signal of 6XHis neither was detected in the conditional medium nor cell lysate from nontransfected HEK $293 \mathrm{~T}$ cells. The cellular concentration of the R5-6/C WT per $2 \times 10^{6}$ cells was $4.16 \pm 1.0 \mathrm{ng} \mathrm{mL}^{-1}$ at $24 \mathrm{~h}$ and $14.4 \pm 1.8 \mathrm{ng} \mathrm{mL}^{-1}$ at $48 \mathrm{~h}$. The amounts in R5-6/C MUT were 8.1 $\pm 1.23 \mathrm{ng} \mathrm{mL}^{-1}$ and $18.33 \pm 3.0 \mathrm{ng} \mathrm{mL}^{-1}$ at $24 \mathrm{~h}$ and $48 \mathrm{~h}$, respectively. The intracellular concentrations of R5-6/C WT were less than R5-6/C MUT after 24h FC=0.51 and $48 \mathrm{~h}$ FC=0.78. The R5-6/CWT levels in the supernatants were $7.36 \pm 1.2 \mathrm{ng} \mathrm{mL}{ }^{-1}$ per $2 \times 10^{6}$ cells after $24 \mathrm{~h}$ and $26.53 \pm 1.6 \mathrm{ng} \mathrm{mL}^{-1}$ after $48 \mathrm{~h}$. In contrast, nothing was detected in the R5-6/C MUT supernatants $24 \mathrm{~h}$ after transfection and its amount after $48 \mathrm{~h}$ was $0.33 \pm 0.57 \mathrm{ng} \mathrm{mL} \mathrm{m}^{-1}$. Difference between supernatant concentration of the R5-6/CWT and the R5-6C/CMUT was significantly obvious after $48 \mathrm{~h}$ (Figure 2C). All data are abstracted in Table 1. 


\section{Discussion}

In this report, we showed that the secretion of Reelin could be altered by S3440P substitution in highly conserved residues of CTR. Learning about the mechanism of Reelin secretion is important not only for the elucidation of its function in brain development but also for understanding the exact role(s) of Reelin in synaptic plasticity in the adult brain (18).

Reelin protein is released by Cajal-Retzus in the marginal zone (MZ) of the brain and is essential for developing neocortex by regulating neuronal cells migration $(1,19)$. It has been shown that the intact CTR is pivotal for the formation of the MZ and development of dendritic cells (20). Homozygous mutations in RELN gene lead to the similar reeler mice phenotype in human including; lissencephaly with cerebellar hypoplasia, ataxia, and cognitive dysfunction (21). Deletion(s) in a part of the eighth RR (RR8) and CTR resulting from retroviral insertion is responsible for reeler phenotype in Orleans strain which is not able to secret Reelin $(15,22)$. The prevention of Reelin secretion in transfected COS-7 cells has been reported by D'Arcangelo et al, as a result of an inserted stop codon in middle of repeat eight of Reelin (RR8) leading to lack of 133 c-terminal residues (4). In contrast, in 1999, Lambert de Rouvroit et al. have shown that a Reelin protein without CTR (32 amino acids) resulting from alternative polyadenylation, could be secreted if overexpressed in COS-7 cells (12). From this results, it could be concluded that Reelin secretion probably requires 100 amino acids which are located between 3328 to 3428 AA. In the present study, releasing a subregion of Human Reelin containing apart of eighth Reelin repeat (3365 to3429) from HEK 293T cells after 24 and 48 hours from transfection bolster this idea that the presence of the predicted 37 residues (3328 to 3365) is not critical for the secretion. 
Here, the substitution of Proline by Serine, at position 3440 of Reelin, caused accumulation of Reelin intracellularly. This might happen because Serine is a polar amino acid, while Proline is a hydrophobic and non-polar amino acid, which is mostly detected buried inside the protein. Also Proline is found in loop regions and gives rigidity to the polypeptide chain cause of imposing certain torsion angles on the protein structure $(23,24)$.

As indicated in Fig 2C, the sum of Reelin mutant in both conditioned medium and cell lysate of R5-6/CMUT were less than the sum of R5-6/CWT after $24 \mathrm{~h}$ and $48 \mathrm{~h}$. As the cloned fragments are expressed under the control of a strong promoter (CMV), modification in translational or post-translational steps and not transcription are expected. No post-translational modifications like glycosylation has been reported for the CTR so far, therefore effects on folding or reducing stability of the folded Reelin protein which also was predicted by in-silico study using IMutant2.0 could be considered to be responsible for these differences. Endoplasmic reticulum (ER) has a main role in packaging and exporting the correctly folded proteins. The ER system is a quality control apparatus that solves intracellular retention of misfolded proteins through degradation of them (25).

Several heterozygous mutations or polymorphisms in $R E L N$ gene have been reported in association with some neurological disorders such as schizophrenia, autism spectrum disorder (ASD), bipolar disorder, and major depression. Additionally, reduced Reelin protein in the brain and blood of these patients have been observed, previously (26-28).Furthermore, a heterozygous mutation, R3441Q, in the CTR has been recognized juxtaposed to our finding substitution in ASD patients (29). 
Although last publications have reported the presence of the CTR is not important for the secretion of Reelin, our results showed that substitution S3440P in this highly conserved residue of the CTR has great effects on Reelin secretion.

\section{Acknowledgments}

We would like to appreciate supports from Ehsan Razmara and Amirreza Bitaraf, Tarbiat Modares University, Tehran, Iran. There is no financial support in this study.

\section{Interest Conflict}

The authors declare that they have not any conflict of interests.

\section{Author contributions}

E.E.GH, M.G, M.M, E.R, A.R.B; Contributed to conception and design. E.E.GH, E.R, A.R.B; Contributed to all experimental work, data and statistical analysis, and interpretation of data. M.G.; Were responsible for overall supervision. E.E.GH, M.G, M.M; Drafted the manuscript, which was revised by M.G and M.M, E.E.GH , E.R, A.R.B All authors read and approved the final manuscript.

\section{References}

1. D'arcangelo G, Miao GG, Chen S-C, Scares HD, Morgan JI, Curran T. A protein related to extracellular matrix proteins deleted in the mouse mutant reeler. Nature 1995; 374(6524): 719. 
2. Verbrugghe $\mathrm{P}$, Bouwer $\mathrm{S}$, Wiltshire $\mathrm{S}$ et al. Impact of the reelin signaling cascade (ligands-receptors-adaptor complex) on cognition in schizophrenia. American Journal of Medical Genetics Part B: Neuropsychiatric Genetics 2012; 159(4): 392-404.

3. Chen Y, Beffert U, Ertunc M et al. Reelin modulates NMDA receptor activity in cortical neurons. Journal of Neuroscience 2005; 25(36): 8209-8216.

4. Fatemi SH. Reelin Glycoprotein: Structure, Biology and Roles in Health and Disease: Springer; 2008.

5. De Bergeyck V, Naerhuyzen B, Goffinet A, de Rouvroit CL. A panel of monoclonal antibodies against reelin, the extracellular matrix protein defective in reeler mutant mice. Journal of neuroscience methods 1998; 82(1): 17-24.

6. He X, Semenov M, Tamai K, Zeng X. LDL receptor-related proteins 5 and 6 in Wnt/ $\beta$ catenin signaling: arrows point the way. Development 2004; 131(8): 1663-1677.

7. Schrijver I, Liu W, Brenn T, Furthmayr H, Francke U. Cysteine substitutions in epidermal growth factor-like domains of fibrillin-1: distinct effects on biochemical and clinical phenotypes. The American Journal of Human Genetics 1999; 65(4): 1007-1020.

8. Utsunomiya-Tate N, Kubo K-i, Tate S-i et al. Reelin molecules assemble together to form a large protein complex, which is inhibited by the function-blocking CR-50 antibody. Proceedings of the National Academy of Sciences 2000; 97(17): 9729-9734.

9. Jossin Y, Ignatova N, Hiesberger T, Herz J, de Rouvroit CL, Goffinet AM. The central fragment of Reelin, generated by proteolytic processing in vivo, is critical to its function during cortical plate development. Journal of Neuroscience 2004; 24(2): 514-521. 
10. Abdolmaleky HM, Cheng Kh, Russo A et al. Hypermethylation of the reelin (RELN) promoter in the brain of schizophrenic patients: a preliminary report. American Journal of Medical Genetics Part B: Neuropsychiatric Genetics 2005; 134(1): 60-66.

11. Royaux I, de Rouvroit CL, D'arcangelo G, Demirov D, Goffinet AM. Genomic Organization of the MouseReelinGene. Genomics 1997; 46(2): 240-250.

12. de Rouvroit CL, Bernier B, Royaux I, de Bergeyck V, Goffinet AM. Evolutionarily conserved, alternative splicing of reelin during brain development. Experimental neurology 1999; 156(2): 229-238.

13. Curry-Hyde A, Chen BJ, Mills JD, Janitz M. Microexons: novel regulators of the transcriptome. Journal of Human Transcriptome 2018; 2(1): 1-6.

14. Nakano Y, Kohno T, Hibi T et al. The extremely conserved C-terminal region of Reelin is not necessary for secretion but is required for efficient activation of downstream signaling. Journal of Biological Chemistry 2007; 282(28): 20544-20552.

15. De Bergeyck V, Nakajima K, de Rouvroit CL et al. A truncated Reelin protein is produced but not secreted in the 'Orleans' reeler mutation (Relnrl-Orl). Molecular brain research 1997; 50(1-2): 85-90.

16. Okoro EU, Zhang H, Guo Z, Yang F, Smith Jr C, Yang H. A subregion of reelin suppresses lipoprotein-induced cholesterol accumulation in macrophages. PloS one $2015 ; 10(8): \mathrm{e} 0136895$.

17. Lammert DB, Middleton FA, Pan J, Olson EC, Howell BW. The de novo autism spectrum disorder RELN R2290C mutation reduces Reelin secretion and increases protein disulfide isomerase expression. Journal of neurochemistry 2017; 142(1): 89-102. 
18. Beffert U, Weeber EJ, Durudas A et al. Modulation of synaptic plasticity and memory by Reelin involves differential splicing of the lipoprotein receptor Apoer2. Neuron 2005; 47(4): 567-579.

19. Ogawa M, Miyata T, Nakajimat K et al. The reeler gene-associated antigen on CajalRetzius neurons is a crucial molecule for laminar organization of cortical neurons. Neuron 1995; 14(5): 899-912.

20. Kohno T, Honda T, Kubo K-i et al. Importance of Reelin C-terminal region in the development and maintenance of the postnatal cerebral cortex and its regulation by specific proteolysis. Journal of Neuroscience 2015; 35(11): 4776-4787.

21. Hong SE, Shugart YY, Huang DT et al. Autosomal recessive lissencephaly with cerebellar hypoplasia is associated with human RELN mutations. Nature genetics 2000; 26(1): 93.

22. Derer P, Derer M, Goffinet A. Axonal secretion of reelin by cajal-retzius cells: Evidence from comparison of normal and RelnOrl mutant mice. Journal of Comparative Neurology 2001; 440(2): 136-143.

23. Krieger F, Möglich A, Kiefhaber T. Effect of proline and glycine residues on dynamics and barriers of loop formation in polypeptide chains. Journal of the American Chemical Society 2005; 127(10): 3346-3352.

24. Karadaghi SA. The 20 Amino Acids and Their Role in Protein Structures. Basics of Protein Structure 2015.

25. Guerriero CJ, Brodsky JL. The delicate balance between secreted protein folding and endoplasmic reticulum-associated degradation in human physiology. Physiological reviews 2012; 92(2): 537-576. 
26. Botella-López A, Burgaya F, Gavín R et al. Reelin expression and glycosylation patterns are altered in Alzheimer's disease. Proceedings of the National Academy of Sciences 2006; 103(14): 5573-5578.

27. Folsom TD, Fatemi SH. The involvement of Reelin in neurodevelopmental disorders. Neuropharmacology 2013; 68: 122-135.

28. Habl G, Schmitt A, Zink M et al. Decreased reelin expression in the left prefrontal cortex (BA9) in chronic schizophrenia patients. Neuropsychobiology 2012; 66(1): 57-62.

29. Lammert DB, Howell BW. RELN mutations in autism spectrum disorder. Frontiers in cellular neuroscience 2016; 10: 84 . 
B.

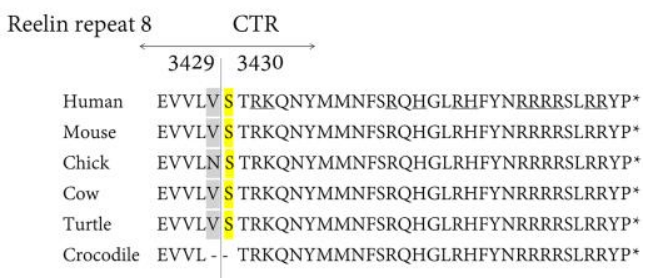

C.

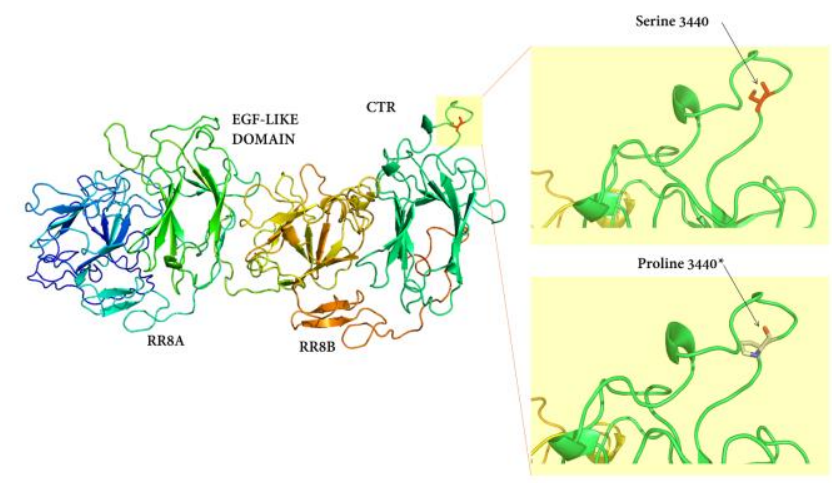

Figure 1. A) Comparison of the CTR region in nucleotide level in various vertebrates. The highlighted rows indicate nucleotides that are not conserved among all of the species listed B) Conservation of the CTR amino acids among different vertebrates. C) Comparison of the normal and the mutated Reelin in the CTR by the predicted structure. Structure modeling of the normal protein and superimposed structure modeling of the mutated protein; the normal and mutated sites of (p. S3440P), is emphasized by a yellow highlight and locally zoomed. 
A.
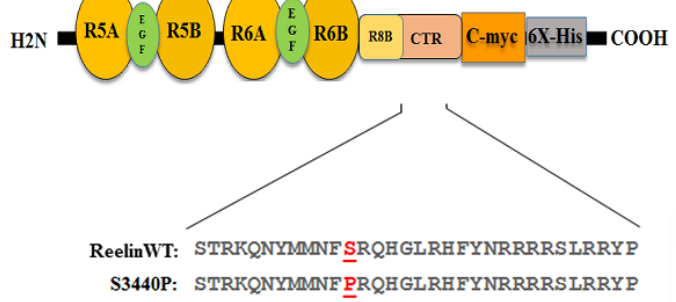

C.

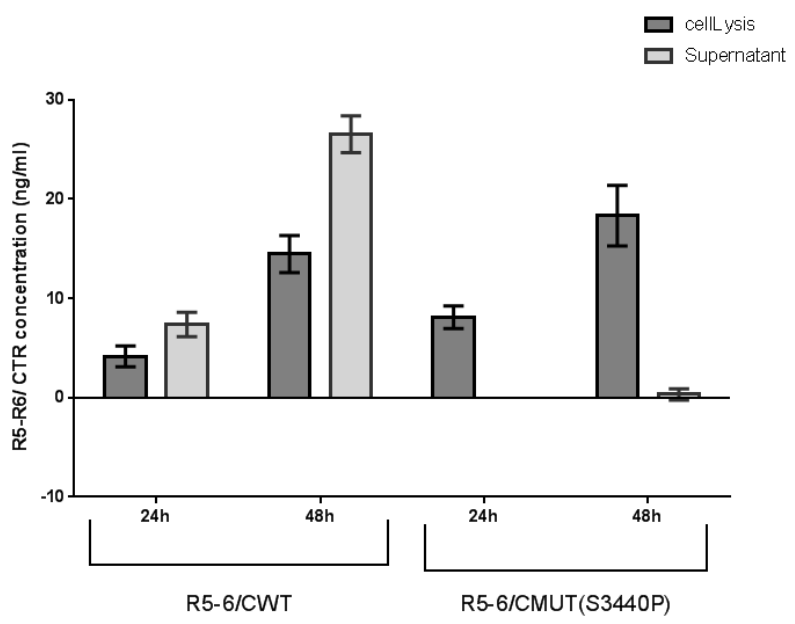

B.
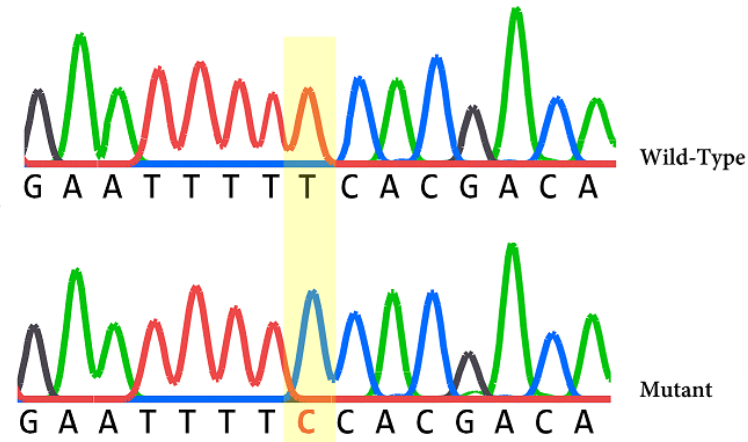

c.10318 T $>$ C, P.S3440P

Figure 2. A) The structure of cloned regions of Reelin. The CTR locates between C-myc and R6B region this region ligated to a part of R8B. The difference between Reelin Wild-type and mutant is indicated in amino acid residues level by red letters. B) Sequence chromatogram showing homozygote state of the nucleotide sequence of $R E L N$ in c.10318 T>C. C) The comparison of the secretion of Reelin between Mutant and wild-type constructs in HEK 293T cells. ( $n=3$, Mean value \pm SD). 
Table 1. Comparison between R5-6 C/ WT and R5-6 C/MUT by using the HEK 293T cells which are transfected by appropriate vectors after 24 and 48 hours follow up.

\begin{tabular}{|c|c|c|c|c|c|c|}
\hline \multirow[t]{2}{*}{ Time (hours) } & \multicolumn{2}{|c|}{ Supernatant } & \multirow[t]{2}{*}{ FC } & \multicolumn{2}{|c|}{ Cell-Lysis } & \multirow[t]{2}{*}{ FC } \\
\hline & R5-6 C/ WT & R5-6 C/ Mut & & R5-6 C/ WT & R5-6 C/Mut & \\
\hline \multirow[t]{2}{*}{24 hours } & $7.36 \pm 1.2 \mathrm{ng}$ & ND & ND & $4.16 \pm 1.04$ & $8.1 \pm 1.23 \mathrm{ng}$ & 0.51 \\
\hline & $\mathrm{mL}^{-1}$ & & & $\mathrm{ng} \mathrm{mL} L^{-1}$ & $\mathrm{~mL}^{-1}$ & \\
\hline \multirow[t]{2}{*}{48 hours } & $26.53 \pm 1.6 \mathrm{ng}$ & $0.33 \pm 0.57 *$ & ND & $14.4 \pm 1.8 \mathrm{ng}$ & $18.33 \pm 3.0$ & 0.78 \\
\hline & $\mathrm{mL}^{-1}$ & $\mathrm{ng} \mathrm{mL}^{-1}$ & & $\mathrm{~mL}^{-1}$ & $n g \mathrm{~mL}^{-1}$ & \\
\hline
\end{tabular}

ND: Not Detectable; * it was out of the detection range of ELISA Kit. FC: Fold Change 